\title{
Superemitters in hybrid photonic systems: A simple lumping rule for the local density of optical states and its breakdown at the unitary limit
}

\author{
Martin Frimmer* and A. Femius Koenderink \\ Center for Nanophotonics, FOM Institute AMOLF, Science Park 104, 1098 XG Amsterdam, The Netherlands
}

(Received 7 April 2012; revised manuscript received 29 November 2012; published 17 December 2012)

\begin{abstract}
We theoretically investigate how the enhancement of the radiative decay rate of a spontaneous emitter provided by coupling to an optical antenna is modified when this "superemitter" is introduced into a complex photonic environment that provides an enhanced local density of optical states (LDOS) itself, such as a microcavity or stratified medium. We show that photonic environments with increased LDOS further boost the performance of antennas that scatter weakly, for which a simple multiplicative LDOS lumping rule holds. In contrast, enhancements provided by antennas close to the unitary limit, i.e., close to the limit of maximally possible scattering strength, are strongly reduced by an enhanced LDOS of the environment. Thus, we identify multiple scattering in hybrid photonic systems as a powerful mechanism for LDOS engineering.
\end{abstract}

DOI: 10.1103/PhysRevB.86.235428

PACS number(s): 78.67.Pt, 42.50.-p

\section{INTRODUCTION}

Optics encompasses the most fascinating part of the electromagnetic spectrum, due to its energetic overlap with electronic transitions in matter. Nanophotonics aims at controlling such transitions by molding light at subwavelength scales. Purcell first predicted that resonators modify the radiative lifetime of spontaneous emitters. ${ }^{1}$ Modern literature discusses the Purcell effect in terms of the local density of optical states (LDOS), a fundamental quantity governing spontaneous emission, thermal radiation, and vacuum forces. ${ }^{2}$ Two tools have emerged to shape the LDOS: On the one hand, interfaces, ${ }^{3}$ photonic crystals, ${ }^{4}$ and dielectric microcavities ${ }^{5}$ modulate the LDOS on length scales of order $\lambda / 2$ via interference. On the other hand, optical antennas ${ }^{6,7}$ employ intrinsic plasmonic resonances to enhance the LDOS on $\lambda / 20$ length scales. Optical antennas are so small compared to the wavelength $\lambda$ that a source-antenna ensemble essentially radiates as a dipole. The term "superemitter" molecule, but with much higher radiative rate. The availability of photonic building blocks on such different length scales raises the exciting idea of integrating deep-subwavelength superemitters in much larger dielectric structures to obtain a combined advantage, for example by placing a nano-antenna inside a microresonator ${ }^{9}$ or onto a planar dielectric antenna. ${ }^{10}$ These developments trigger the question of how the LDOS of such hybrid systems emerges from that of the separate entities. Nano-optic devices can be interpreted as lumped optical elements, ${ }^{11,12}$ in analogy to lumped electrical circuit elements. Since in this analogy the LDOS acts as an impedance for spontaneous emitters, some circuit rule might be hoped for, which lumps the LDOS provided by each photonic building block individually to yield the LDOS of the hybrid architecture.

This paper investigates how the decay rate enhancement provided by a superemitter varies when it is placed within a larger photonic system, i.e., how the LDOS lumps. We illustrate our theory, which is valid for any background LDOS, by two archetypical examples: First, we investigate a superemitter weakly coupled to high- $Q$ resonances. Second, we consider a superemitter in front of a perfect mirror without any resonances. Our analytic, yet fully electrodynamic model yields a simple multiplicative LDOS lumping rule for moderate antenna factors. For strongly scattering antennas, i.e., antennas close to the unitary limit, this simple lumping rule breaks down and the total enhancement becomes proportional to the inverse LDOS of the background system. This insight paves the way for engineering the LDOS by exploiting multiple scattering in hybrid photonic systems.

\section{THE SUPEREMITTER CONCEPT}

The radiative decay rate enhancement of a quantum emitter can be calculated via the power required to sustain a monochromatic classical point current $\boldsymbol{j}=\dot{\boldsymbol{p}}_{0}$ that loses energy by radiation. ${ }^{2}$ This power equals the cycle averaged work per unit time done by the source's electric field on its own dipole moment $\boldsymbol{p}_{0}$. The electric field generated at position $\boldsymbol{r}$ due to a source $\boldsymbol{p}_{0}$ at $\boldsymbol{r}_{0}$ is calculated via the electric Green function $\boldsymbol{G}\left(\boldsymbol{r}, \boldsymbol{r}_{0}\right)$ of the respective system. This yields the power required to drive the source $P=\frac{1}{2} \omega \boldsymbol{p}_{0}^{T} \operatorname{Im} \boldsymbol{G}\left(\boldsymbol{r}_{0}, \boldsymbol{r}_{0}\right) \boldsymbol{p}_{0}$. With LDOS we refer to the (scalar) projection of $\operatorname{Im} G$ on a unit vector along $\boldsymbol{p}_{0}$, i.e., to the decay rate modification of a molecular dipole $\boldsymbol{p}_{0}$ at $\boldsymbol{r}_{0}$. Every strategy to boost decay rates via a photonic structure, be it large and dielectric, or a nano-antenna, represents a modification of $\operatorname{Im} \boldsymbol{G}$, which comprises all losses due to the environment. These losses include radiation into the continuum, generation of polaritons, e.g., surface or particle plasmons, as well as quenching due to Ohmic losses. Rather than considering its contribution to $\operatorname{Im} \boldsymbol{G}$, the small size and dipolar nature of a nano-antenna, however, suggest to interpret its rate enhancement as an enhancement of the dipole moment $\boldsymbol{p}_{0} .{ }^{8}$ The simplest optical antenna is just a particle with polarizability tensor $\alpha(\omega){ }^{6}$ At distance $d$ in the near field of an emitter, the particle acquires a large dipole moment $\propto \alpha / d^{3}$. The total dipole moment of the emitter-particle assembly $\boldsymbol{p}=\left[1+\boldsymbol{\alpha} / d^{3}\right] \boldsymbol{p}_{0}$ can exceed by far the source's dipole moment $\boldsymbol{p}_{0}$, rationalizing the term "superemitter". If $d \ll \lambda$, the power radiated by the superemitter 8,10

$$
P=\frac{1}{2} \omega\left|\boldsymbol{p}_{0}\right|^{2} \operatorname{LDOS}_{\mathrm{B}}\left(\boldsymbol{r}_{0}\right) \times A
$$

exceeds that of the bare source by the antenna factor $A=$ $\left|1+\alpha / d^{3}\right|^{2} \approx|\alpha|^{2} / d^{6} .{ }^{13}$ The rate enhancement provided by 
the embedding background is described by $\operatorname{LDOS}_{\mathrm{B}}$. Mie calculations show that $A$ accurately describes antenna particles up to $60 \mathrm{~nm}$ in diameter in vacuum. ${ }^{14}$ This reasoning suggests that a superemitter can simply replace a bare molecule as a probe of larger photonic systems, since a simple product rule lumps the enhancements provided by antenna and photonic environment as $A \times \operatorname{LDOS}_{\mathrm{B}}$.

\section{ANALYTIC POINT SCATTERING MODEL}

We analyze the hypothesis of a simple lumping rule in an analytic electrodynamic point scattering theory, which is exact to all multiple scattering orders, with the sole assumption that the scatterers that constitute the antenna are well approximated as point dipoles. The $N$ scatterers acquire dipole moments $\boldsymbol{p}_{1}, \ldots, \boldsymbol{p}_{N}$ in proportion to their polarizabilities $\boldsymbol{\alpha}_{1}, \ldots, \boldsymbol{\alpha}_{N}$, and the electric fields $\boldsymbol{E}\left(\boldsymbol{r}_{1}\right), \ldots, \boldsymbol{E}\left(\boldsymbol{r}_{N}\right)$ at their locations $\boldsymbol{r}_{n}$ through $^{15}$

$$
\boldsymbol{p}_{n}=\boldsymbol{\alpha}_{n}\left[\boldsymbol{E}_{\mathrm{in}}\left(\boldsymbol{r}_{n}\right)+\sum_{m \neq n} \boldsymbol{G}_{\mathrm{B}}\left(\boldsymbol{r}_{n}, \boldsymbol{r}_{m}\right) \cdot \boldsymbol{p}_{m}\right] .
$$

By construction, the antenna described by the $\boldsymbol{\alpha}_{n}$ is explicitly separated from the background that it probes, which is quantified by its Green function $\boldsymbol{G}_{\mathrm{B}}$. For a consistent theory, three facts need to be accounted for. First, the particle polarizability directly depends on the background via ${ }^{12}$

$$
\boldsymbol{\alpha}_{n}^{-1}=\boldsymbol{\alpha}_{n, 0}^{-1}-\boldsymbol{G}_{\mathrm{B}}\left(\boldsymbol{r}_{n}, \boldsymbol{r}_{n}\right),
$$

where $\boldsymbol{\alpha}_{n, 0}$ is the electrostatic polarizability. Isotropic particles can acquire anisotropy due to anisotropy in radiation damping given by $\operatorname{Im} \boldsymbol{G}_{\mathrm{B}}$ and resonance shift due to $\operatorname{Re} \boldsymbol{G}_{\mathrm{B}}$ in complex photonic systems. ${ }^{16}$ Second, the source in our model is a single (unpolarizable) dipole $\boldsymbol{p}_{0}$ at $\boldsymbol{r}_{0}$ so $\boldsymbol{E}_{\text {in }}\left(\boldsymbol{r}_{n}\right)=\boldsymbol{G}_{\mathrm{B}}\left(\boldsymbol{r}_{n}, \boldsymbol{r}_{0}\right) \boldsymbol{p}_{0}$. The description of a quantum emitter as a constant-current source is valid as long as Fermi's golden rule holds, i.e., far from the regime of strong coupling between source and environment. ${ }^{17}$ Regarding the coupling strength between the optical antenna and the background system we stress that our formalism Eq. (2) holds both in the weak and in the strong coupling regimes since it contains full electrodynamic interactions to all scattering orders. However, we note that this work remains entirely in the realm of weak coupling. Third, the total decay rate of the source is found via the cycle-averaged work done by the total electric field on the source $\boldsymbol{p}_{0} \cdot{ }^{2}$ Therefore the LDOS equals

$\boldsymbol{p}_{0}^{T} \cdot \operatorname{Im} \boldsymbol{G}_{B}\left(\boldsymbol{r}_{0}, \boldsymbol{r}_{0}\right) \cdot \boldsymbol{p}_{0}+\operatorname{Im} \sum_{n \geqslant 1} \boldsymbol{p}_{0}^{T} \cdot \boldsymbol{G}_{\mathrm{B}}\left(\boldsymbol{r}_{0}, \boldsymbol{r}_{n}\right) \cdot \boldsymbol{p}_{n}$.

The first term is the $\operatorname{LDOS}_{\mathrm{B}}$ provided by the background without antenna, while the second term arises from the antenna. We calculate the LDOS of the hybrid system from Eq. (4) after solving Eq. (2) for the $3 N$ dipole moments $\boldsymbol{p}_{1}, \ldots, \boldsymbol{p}_{N}$. We use the exact Green function for a sphere ${ }^{18}$ and a planar interface ${ }^{2,19}$ to evaluate how a superemitter probes the two canonical cases of microcavity resonances and nonresonant interfaces. ${ }^{3}$

\section{A SUPEREMITTER COUPLED TO A MICROCAVITY}

As a superemitter we consider a source $\boldsymbol{p}_{0}$ in the gap of a dimer antenna with $\boldsymbol{p}_{0}$ along the symmetry axis [see Fig. 1(a)]. The $40 \mathrm{~nm}$ diameter $\mathrm{Ag}$ spheres forming the dimer have a center-center distance of $60 \mathrm{~nm} .{ }^{20}$ The dashed line in Fig. 1(b) shows Im $\boldsymbol{\alpha}$ of a single antenna particle in vacuum. The decay rate enhancement of the source in the dimer-antenna gap [solid line in Fig. 1(b)] reaches about 1200, in good agreement with full multipole calculations. ${ }^{21}$ The dimer resonance is redshifted and broadened by super-radiant damping compared to the single particle due to longitudinal symmetric dipolar plasmon hybridization. ${ }^{22}$ Positioning the emitter in the center of the dimer maintains a safe distance to the metal particles, keeping Ohmic quenching losses to a minimum while offering significant enhancement at a quantum efficiency of the superemitter exceeding $90 \%$.

As a first test of LDOS lumping we consider as a background a glass sphere ( $n=1.5$, radius $1.2 \mu \mathrm{m})$ supporting whispering-gallery modes, illustrated in Fig. 1(a). Characteristic resonances are clearly visible as sharp peaks with $Q \approx 700$ in the sphere's extinction efficiency ${ }^{23}$ [Fig. 1(c), dashed line]. The Purcell factor for a radially oriented source $50 \mathrm{~nm}$ from the sphere surface [solid line in Fig. 1(c)] reaches moderate values around 10 . Only every second peak in extinction yields Purcell enhancement, which reflects the field orientation according to the common TE/TM type classification. ${ }^{24}$ We now place the superemitter with the center of the closest antenna particle $50 \mathrm{~nm}$ from the sphere's surface and the symmetry axis pointing radially outwards [see Fig. 1(a)]. We stress that throughout the spectrum all couplings between source, antenna, and cavity are weak. To determine the regime of coupling, the coupling

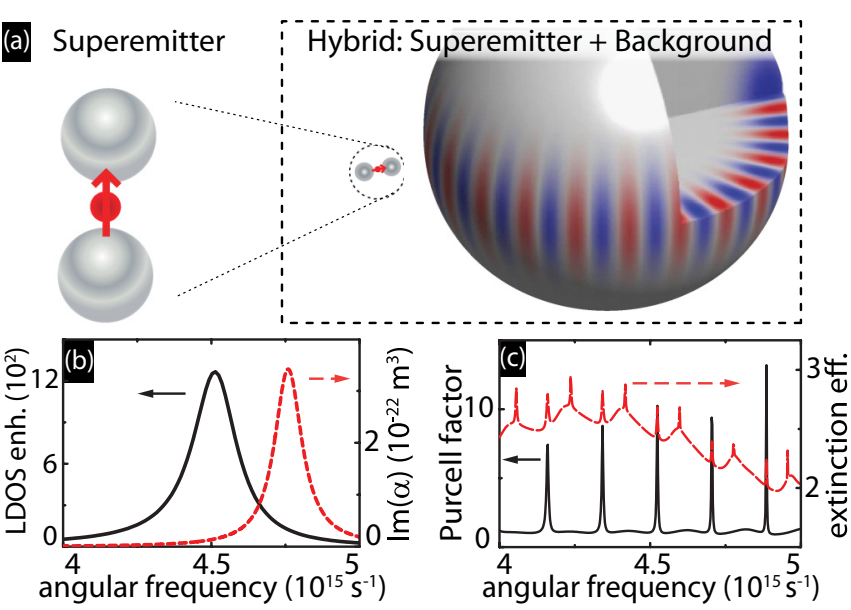

FIG. 1. (Color online) (a) Left: Sketch of a superemitter formed by two silver spheres. The fluorescent source is located between the two particles with its dipole moment along the symmetry axis. Right: Hybrid photonic system composed of a superemitter embedded in a background system formed by a dielectric sphere. Red and blue colors illustrate fields of a whispering-gallery mode. (b) Dashed line: polarizability of a single antenna particle in vacuum. Solid line: antenna enhancement factor for the superemitter sketched in (a) in vacuum. (c) Dashed line: Extinction efficiency of a dielectric sphere showing narrow Mie resonances. Solid line: Purcell factor of the Mie sphere $50 \mathrm{~nm}$ from its surface. 

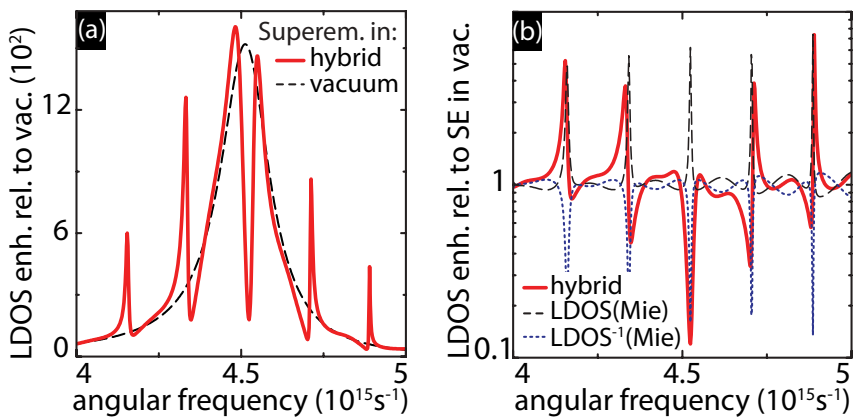

FIG. 2. (Color online) (a) Solid line (hybrid system): Decay rate of the source in the gap of the superemitter next to the Mie sphere normalized to the rate of the source in vacuum. Dashed line (superemitter in vacuum): Decay rate of the source in the gap of the superemitter in vacuum normalized to the rate of the source in vacuum. At the Mie resonances, the hybrid LDOS is drastically modified compared to that of the antenna in vacuum. (b) Solid line (hybrid system): Decay rate enhancement in hybrid system [solid line in (a)], normalized to the rate enhancement in the superemitter in vacuum [dashed line in (a)]. Off antenna resonance, the superemitter benefits from the LDOS enhancement offered by the sphere (dashed line), while on antenna resonance the enhancement is suppressed by the inverse of the sphere's LDOS (dotted line).

strength between resonators has to be measured against their respective damping rates. ${ }^{25}$ For a fluorescent source (damping $\gamma_{\mathrm{s}} \approx \mathrm{ns}^{-1}$ ) typically $\gamma_{\mathrm{ant}} \approx 10^{6} \gamma_{\mathrm{s}} \approx \mathrm{fs}^{-1}$ at a Purcell factor $\mathcal{F}_{\mathrm{P}}=\frac{4|g|^{2}}{\gamma_{\text {ant }} \gamma_{\mathrm{s}}} \approx 1200$, such that the coupling $g \ll \gamma_{\text {ant }}$. Similar arguments hold for the antenna-cavity and cavity-source coupling strengths. Accordingly, the antenna does not significantly shift or spoil the microsphere resonances, as also predicted by Waldron's formula $\Delta \omega / \omega=-(\alpha / \mathcal{V})|E|^{2}$, due to their large mode volumes $\mathcal{V}$ compared to $\boldsymbol{\alpha}$, their moderate $Q$ factors, ${ }^{24,26}$ and placement of the antenna far from the normalized mode function $|E|^{2}$ maxima. The solid line in Fig. 2(a) is the decay rate enhancement for the emitter embedded in the antenna, in turn located next to the Mie sphere, i.e., the rate in the hybrid system normalized to the rate of the bare source in vacuum. While the overall shape of the enhancement provided by the antenna in vacuum [Fig. 2(a), dashed] is still visible, sharp features appear at five spectral positions coinciding with the sphere's whispering-gallery modes [cf. Fig. 1(c)]. To illustrate the effect of the background system on the superemitter we normalize the decay rate enhancement in the hybrid system [solid line in Fig. 2(a)] to the enhancement provided by the bare antenna in vacuum [dashed line in Fig. 2(a)] and plot it as the solid line in Fig. 2(b). The sharp enhancements in the wings of the antenna resonance follow the LDOS of the sphere, denoted by the dashed line in Fig. 2(b). Therefore, off antenna resonance, at still significant antenna factors, we find the anticipated behavior of a superemitter that the already antenna-enhanced decay rate is further boosted by a high $\operatorname{LDOS}_{\mathrm{B}}$ of the background. Furthermore, we note the dispersive features in the enhancement which swap orientation upon crossing the antenna resonance. Surprisingly, however, on antenna resonance, the LDOS enhancement is strongly suppressed by the Mie sphere. This LDOS suppression close to antenna resonance cannot be explained by a spoiling or detuning of the cavity by the antenna, since this would only result in a shift or absence of a sharp line of extra enhancement on top of the bare antenna factor. Since Waldron's formula excludes that the LDOS suppression results from a spoiling of the Mie resonances by the antenna, Fig. 2(a) hence implies a spoiling of the antenna by the cavity resonance.

To interpret Eq. (1) correctly, the scattering strength of the antenna needs to be taken into account, which is given by the scatterer's optical volume, i.e., its polarizability, measured against the inverse background LDOS according to Eq. (3). To illustrate the effect of the background LDOS on the scattering strength of an optical antenna, we now discuss an antenna significantly smaller than the wavelength and described as a single scatterer with polarizability $\boldsymbol{\alpha} .^{27}$ Equation (3) ensures that the optical theorem $\operatorname{Im} \alpha \geqslant \operatorname{LDOS}_{\mathrm{B}}|\alpha|^{2}$ is fulfilled, where equality holds for the case of no material loss. ${ }^{28}$ Thereby Eq. (3) strictly bounds the polarizability according to

$$
\operatorname{Im}(\boldsymbol{\alpha}) \leqslant \frac{1}{\operatorname{Im} \boldsymbol{G}_{\mathrm{B}}},
$$

which is a general form of the unitary limit. This limit is a well known fundamental constraint in any scattering theory. ${ }^{29}$ In vacuum, the unitary limit for extinction cross section is $\sigma_{\text {ext }}=4 \pi k \operatorname{Im} \alpha \leqslant \frac{3}{2 \pi} \lambda^{2}$, a limit reached by an ideal scatterer on resonance, and closely approached by any plasmon particle above $20 \mathrm{~nm}$ in size. ${ }^{14}$ The unitary limit Eq. (5) states that $\boldsymbol{\alpha}$ of a strong scatterer, and hence the dipole moment it acquires, is proportional to the inverse LDOS. Since a strong scatterer is predominantly damped by radiation, increasing the background LDOS increases its loss and therefore suppresses the scatterer's response. ${ }^{30}$

To quantitatively verify that the unitary limit indeed governs the hybrid system's LDOS we evaluate Eq. (4) for a physically small superemitter. The hybrid system's LDOS is then governed by the LDOS enhancement provided by the optical antenna $\operatorname{LDOS}_{\mathrm{A}}$ which is dominated by $\operatorname{Re} \boldsymbol{G}_{\text {vac }}$ to read

$$
\mathrm{LDOS}_{\mathrm{A}}=\boldsymbol{p}_{0}^{T} \operatorname{Re} \boldsymbol{G}_{\mathrm{vac}}\left(\boldsymbol{r}_{0}, \boldsymbol{r}_{1}\right) \operatorname{Im} \boldsymbol{\alpha} \operatorname{Re} \boldsymbol{G}_{\mathrm{vac}}\left(\boldsymbol{r}_{1}, \boldsymbol{r}_{0}\right) \boldsymbol{p}_{0},
$$

which is of order $1 / d^{6}$ (with $d=\left|\boldsymbol{r}_{1}-\boldsymbol{r}_{0}\right|$ ) and precisely yields Eq. (1). ${ }^{31}$ To illustrate the effect of the background system on Im $\alpha_{\text {Mie }}$ we plot in Fig. 3(a) the radial component of the sphere's Green function $\boldsymbol{G}_{\mathrm{Mie}}$ at the superemitter $(50 \mathrm{~nm}$ from the sphere surface). Real and imaginary parts of $\boldsymbol{G}_{\mathrm{Mie}}$ show the typical lineshape of a resonance, where $\operatorname{Im} \boldsymbol{G}_{\text {Mie }}$ by definition equals the microcavity Purcell factor at the source [cf. Fig. 1(c)]. In Fig. 3(b) we plot as the solid line the radial component of $\operatorname{Im} \alpha_{\text {Mie }}$ of an antenna located $50 \mathrm{~nm}$ from the sphere surface. The values of $\boldsymbol{G}_{\text {Mie }}$ in Fig. 3(a) are the correction terms entering Eq. (3) that modify $\boldsymbol{\alpha}_{\text {Mie }}$ close to the sphere [solid line in Fig. 3(b)] with respect to $\boldsymbol{\alpha}_{\mathrm{vac}}$ in vacuum [dash-dotted line in Fig. 3(b)]. Close to antenna resonance $\operatorname{Im} \alpha_{\mathrm{Mie}}$ is indeed limited by the inverse of the sphere's LDOS [Fig. 3(b), dotted line]. The transition from enhancement to inhibition in the lumped LDOS going from weak to strong scattering is captured by amending the antenna factor $A$ in Eq. (1) with Eq. (3), such that when neglecting the real frequency shift the radiated power reads

$$
P \propto\left|\boldsymbol{\alpha}_{0}^{-1}-i \operatorname{LDOS}_{\mathrm{B}}\right|^{-2} \operatorname{LDOS}_{\mathrm{B}} .
$$



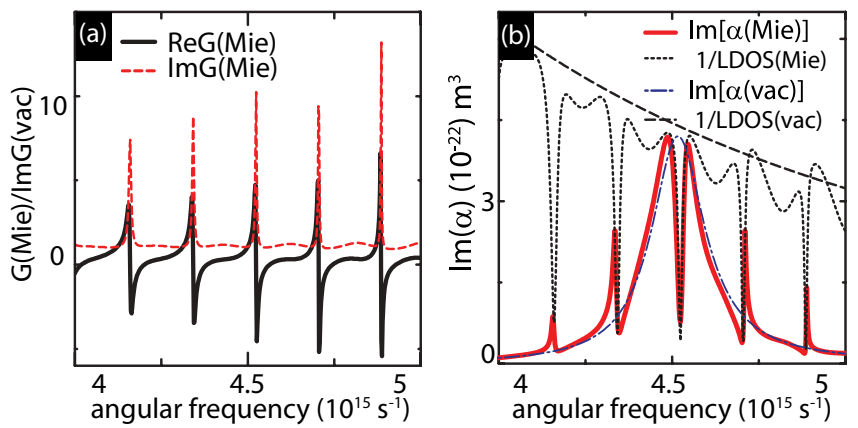

FIG. 3. (Color online) (a) Real (solid) and imaginary (dashed) parts of the radial component of the Green function of the Mie sphere $50 \mathrm{~nm}$ from sphere's surface. These terms enter the radiation correction to $\boldsymbol{\alpha}_{\mathrm{Mie}}$. (b) Solid line: Radial component of the antenna polarizability $\boldsymbol{\alpha}_{\text {Mie }}$ when located $50 \mathrm{~nm}$ from the Mie sphere. Dash dotted line: $\boldsymbol{\alpha}_{\text {vac }}$ for the same antenna in vacuum. While $\boldsymbol{\alpha}_{\text {vac }}$ is limited by the inverse vacuum LDOS (dashed line), $\boldsymbol{\alpha}_{\mathrm{Mie}}$ is bounded by the inverse of the sphere's LDOS (dotted line), which leads to the suppression of $\boldsymbol{\alpha}_{\text {Mie }}$ close to the antenna resonance.

For small $\boldsymbol{\alpha}_{0}$, i.e., in the limit of weak scattering, $P \propto$ $\left|\boldsymbol{\alpha}_{0}\right|^{2} \mathrm{LDOS}_{\mathrm{B}}$ since weak Rayleigh scatterers are constant current sources unaffected by the unitary limit. ${ }^{32}$ Therefore, for weak scatterers a simple multiplicative lumping rule holds. In the limit of strong scattering $P \propto \operatorname{LDOS}_{\mathrm{B}}^{-1}$, since a scatterer at the unitary limit is not a constant current source. ${ }^{33}$ Accordingly, close to antenna resonance, the enhancement of the hybrid system [solid line in Fig. 2(b)] follows the inverse of the Mie sphere's LDOS.

\section{SUPEREMITTER AND CAVITY AS COUPLED HARMONIC OSCILLATORS}

The hybrid system LDOS in Fig. 2(a) closely follows Fano lineshapes that occur generally in coupled-harmonicoscillator models ${ }^{34}$ that describe, for example, electromagnetically induced transparency (EIT) in quantum optics ${ }^{35}$ and plasmonics. ${ }^{36}$ With the surge of plasmonic Fano resonances ${ }^{36}$ a coupled-oscillator simile, initially put forward by Alzar et al., has emerged as the prime model in order to fit and discuss lineshapes of various observables that arise due to the interaction of several photonic constituents. ${ }^{34}$ It is an open question in nanophotonics under which circumstances a mechanical-oscillator model can be applied to describe strongly scattering systems and how it relates to a fully electrodynamic model such as our coupled-dipole formalism. We therefore treat the optical antenna coupled to a single cavity mode explicitly as coupled oscillators to identify the similarities and differences with a coupled-mechanicaloscillator model.

Alzar et al. consider two harmonic oscillators, whose positions are described by spatial coordinates $x_{1,2}$, characteristic frequencies $\omega_{1,2}$, and dissipative damping rates $\gamma_{1,2}$ coupled at a rate $\kappa$ and the driving forces (normalized by the respective masses) are $F_{1,2}$, such that the equations of motion read

$$
\ddot{x}_{1,2}+\gamma_{1,2} \dot{x}_{1,2}+\omega_{1,2}^{2} x_{1,2}+\kappa^{2} x_{2,1}=F_{1,2} \text {. }
$$

Assuming a harmonic time dependence and $F_{2}=0$ the response of the first oscillator to a driving force $F_{1}$ is

$$
x_{1}=\chi_{1} F_{1}=\frac{1}{\omega_{1}^{2}-\omega^{2}-i \omega \gamma_{1}+\frac{\kappa^{4}}{\omega_{2}^{2}-\omega^{2}-i \omega \gamma_{2}}} F_{1} \text {, }
$$

where we have defined the mechanical response function $\chi_{1}$. To quantitatively relate to the coupled-dipole model we need to define an observable in the mechanical model that represents the LDOS. Associating the driven oscillator with the antenna a natural choice is to consider the work done by the driving force $F_{1}$ on oscillator $x_{1}$,

$$
W_{1}=\left\langle\operatorname{Re} F_{1} \cdot \operatorname{Re} x_{1}\right\rangle=F_{1}^{\dagger} \operatorname{Im} \chi_{1}(\omega) F_{1} .
$$

The mechanical driving force $F_{1}$ then corresponds to the electric field generated by the molecular source driving the antenna $\operatorname{Re} \boldsymbol{G} \boldsymbol{p}_{0}$, and we obtain one-to-one correspondence between the antenna factor Eq. (6) and the mechanicaloscillator model Eq. (10) if two conditions are satisfied. First, the multiple scattering problem of an antenna in a complex background system can only be described in a coupled-oscillator framework with a single driven oscillator if the antenna is small enough such that it is sufficient to consider the leading term of order $d^{-6}$ that led to Eq. (6). Second, we still need to quantitatively map the corrected polarizability $\alpha$ onto the mechanical response function $\chi_{1}$. To this end we consider a classical scatterer coupled to a single mode of a cavity. ${ }^{37}$ It turns out that electromagnetism imposes two strong constraints on the parameters of Eq. (8). First, the damping constant $\gamma_{1}$ associated with the antenna, besides accounting for possible Ohmic losses, must contain the scatterer's volume $V$ measured against the unitary limit such that $\gamma_{1}=\omega_{1} V \operatorname{LDOS}_{\mathrm{vac}}$. This constraint ensures that radiation damping of the antenna due to coupling to the radiative continuum modes is included in the oscillator model Eq. (8). Second, also the coupling constant $\kappa$ contains the scatterer's volume measured against the unitary limit and the cavity mode volume $\mathcal{V}$, such that $\kappa^{4}=\omega_{1}^{4} \frac{\mathcal{F}_{\mathrm{P}}}{Q} \operatorname{LDOS}_{\text {vac }} V$, with the Purcell factor $\mathcal{F}_{\mathrm{P}}=\frac{3}{4 \pi^{2}} \lambda^{3} \frac{Q}{\mathcal{V}}$. Therefore, while naturally included in a consistent electrodynamic model, radiation effects have to enter a mechanical-oscillator model as a deus ex machina to contain the unitary limit by constraining both the coupling constant $\kappa$ and the damping rate $\gamma_{1}$ in Eq. (8). Any other choice of $\kappa$ and $\gamma_{1}$ would violate energy conservation by neglecting coupling to the radiative continuum. That the unitary limit is not by itself a consequence of classical-oscillator models reflects the essential fact that, unlike mechanical oscillators, optical resonances are superradiant or subradiant depending on interference in the radiative continuum.

\section{A SUPEREMITTER IN FRONT OF A MIRROR}

While it is highly promising that hybrid systems allow engineering of LDOS using the toolbox of coupled-oscillator systems, the physics of hybrid photonic systems is far more general than that of coupled oscillators alone. Indeed, the surprising spoiling of the antenna enhancement generally occurs for any large background $\mathrm{LDOS}_{\mathrm{B}}$, also in absence of any resonance. As one example of the general validity of Eq. (7) beyond microcavity resonances, we examine a 


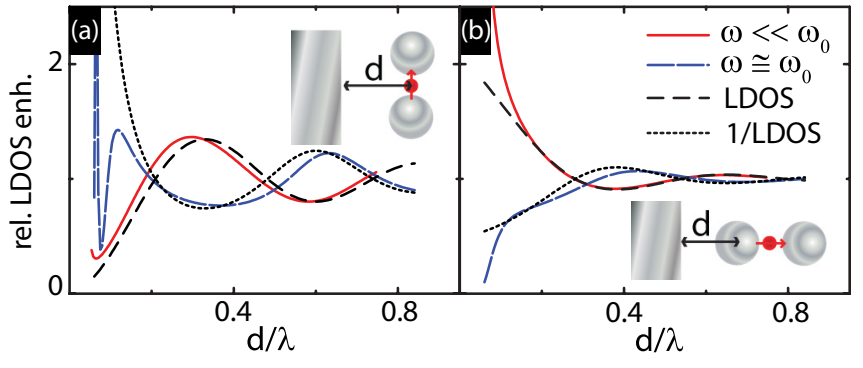

FIG. 4. (Color online) Relative LDOS enhancement for the superemitter as a function of distance to a near-perfect mirror. (a) Superemitter oriented parallel to the mirror surface (see inset). Solid line: A superemitter with source far below antenna resonance $\left[\omega=4.0 \times 10^{15} \mathrm{~s}^{-1}\right.$, cf. Fig. 1(b)] traces the LDOS of the mirror (dashed line), except at small superemitter-mirror separations. A source close to antenna resonance (long dashed line, $\omega=4.5 \times$ $10^{15} \mathrm{~s}^{-1}$ ) traces the inverse of the mirror LDOS (dotted line), except at small separations. (b) Same as (a) for the superemitter perpendicular to the mirror (see inset).

near-perfect mirror $(\epsilon=-200)$ as a background that modifies the LDOS without any resonances. ${ }^{3}$ In Fig. 4(a), we plot the decay rate of the superemitter in front of the mirror normalized to the superemitter in vacuum as a function of distance to the mirror. The antenna axis is parallel to the mirror (see sketch in inset). Far below antenna resonance, the enhancement [Fig. 4(a), solid line] follows the mirror LDOS (dashed line) as expected from the multiplicative LDOS lumping rule. Close to antenna resonance the antenna is close to the unitary limit and therefore the total rate enhancement follows the inverse of the mirror LDOS. At intermediate frequencies we observe a smooth transition between the two cases illustrated in Fig. 4 (data not shown). For the superemitter oriented perpendicularly to the mirror [Fig. 4(b)] we observe the analog behavior as for the parallel case. The product lumping rule and its crossover to inverse proportionality are hence generic, and only break down for small superemitter-mirror separations (ca. $0.2 \lambda$ in Fig. 4) where the small-size approximation leading to Eq. (7) breaks down. When the superemitter size is comparable to its distance to its own mirror image, superemitters also sense gradients in $\boldsymbol{G}$. While outside the simple lumping rule Eq. (7), this exciting regime, fully contained in our general formalism Eq. (2), is of great interest as a tailorable analogon to recent experiments on probing multipolar LDOS. ${ }^{38}$

\section{CONCLUSIONS}

In conclusion, we have examined how the LDOS inside a superemitter probes the LDOS of a complex photonic environment. Generally, for any superemitter with a moderate antenna factor the LDOS enhancements of antenna and background multiply and a small superemitter will serve as an LDOS probe for a large background system, exactly as the term suggests. ${ }^{8}$ In surprising contrast, a superemitter with an antenna at the unitary limit probes the inverse background LDOS, since increasing radiation damping reduces the polarizability of strong scatterers. ${ }^{29}$ Our findings imply that if a general lumping rule for optical source impedances, ${ }^{12}$ i.e., LDOS, can be found, it must take into account not just the bare superemitter LDOS, but also how close the antenna is to the unitary limit. While throughout this work antenna and background were only weakly coupled, our formalism is equally applicable to the strong-coupling case where the presence of the antenna significantly spoils the cavity resonances. Regarding source-environment coupling strength, our antenna-cavity hybrid offers yet another exciting prospect: Off antenna resonance but on a cavity resonance, the Purcell enhancement of the hybrid reaches values as high as $\mathcal{F}_{\mathrm{P}} \approx 1000$ at a quality factor comparable to that of the cavity. These parameters might suffice to bring a typical quantum $\operatorname{dot}^{8}$ with $\gamma_{\mathrm{QD}} \approx(10 \mathrm{~ns})^{-1}$ into the strong-coupling regimenotably with robust building blocks whose individual Purcell factors are an order of magnitude lower than that of the hybrid. In addition, our insights bear a plethora of exciting prospects for LDOS engineering in many background systems beyond cavity resonances, including plasmonics, indefinite media and metamaterials, and van Hove singularities at photonic-bandgap edges. Embedding complex antennas with beyond-dipole character ${ }^{39}$ into such systems could provide yet another route for LDOS engineering. One might consider nanomanipulative switching of superemitters by moving them with respect to high- $Q$ resonators. ${ }^{40}$ Furthermore, our work could lead the quest for ultrastrong optical antennas towards counterintuitive hybrids of nanoantennas embedded in photonic bandgap devices. As a further implication, our findings shed new light on attempts to use a scatterer as a broadband probe of thermally populated modes, since the scatterer acquires spectral features thanks to the environment that it probes. ${ }^{41}$ Notably, hybrid photonic systems might benefit from large field enhancements inside the antenna that occur with convenient moderate- $Q$ cavities, an exciting prospect for single-molecule detection. ${ }^{42}$

\section{ACKNOWLEDGMENTS}

This work is part of the research program of the "Stichting voor Fundamenteel Onderzoek der Materie (FOM)", which is financially supported by the "Nederlandse Organisatie voor Wetenschappelijk Onderzoek (NWO)". A.F.K. gratefully acknowledges an NWO-Vidi grant for financial support.

\footnotetext{
*frimmer@amolf.nl

${ }^{1}$ E. M. Purcell, Phys. Rev. 69, 681 (1946).

${ }^{2}$ L. Novotny and B. Hecht, Principles of Nano-Optics (Cambridge University Press, Cambridge, UK, 2006).

${ }^{3}$ K. H. Drexhage, J. Lumin. 1, 693 (1970); E. Snoeks, A. Lagendijk, and A. Polman, Phys. Rev. Lett. 74, 2459 (1995).
}

${ }^{4}$ P. Lodahl, A. F. van Driel, I. S. Nikolaev, A. Irman, K. Overgaag, D. Vanmaekelbergh, and W. L. Vos, Nature (London) 430, 654 (2004); M. Barth, N. Nüsse, B. Löchel, and O. Benson, Opt. Lett. 34, 1108 (2009).

${ }^{5}$ Optical Microcavities, edited by K. Vahala, Advanced Series in Applied Physics, Vol. 5 (World Scientific, Singapore, 2005). 
${ }^{6}$ P. Anger, P. Bharadwaj, and L. Novotny, Phys. Rev. Lett. 96, 113002 (2006); S. Kühn, U. Håkanson, L. Rogobete, and V. Sandoghdar, ibid. 97, 017402 (2006).

${ }^{7}$ S. Schietinger, M. Barth, T. Aichele, and O. Benson, Nano Lett. 9, 1694 (2009); A. Kinkhabwala, Z. Yu, S. Fan, Y. Avlasevich, K. Müllen, and W. E. Moerner, Nat. Photon. 3, 654 (2009).

${ }^{8}$ J. N. Farahani, D. W. Pohl, H.-J. Eisler, and B. Hecht, Phys. Rev. Lett. 95, 017402 (2005).

${ }^{9}$ O. Benson, Nature (London) 480, 193 (2011).

${ }^{10}$ X.-W. Chen, M. Agio, and V. Sandoghdar, Phys. Rev. Lett. 108, 233001 (2012).

${ }^{11}$ N. Engheta, A. Salandrino, and A. Alù, Phys. Rev. Lett. 95, 095504 (2005); N. Engheta, Science 317, 1698 (2007); A. Alù and N. Engheta, Phys. Rev. B 78, 195111 (2008); Phys. Rev. Lett. 101, 043901 (2008).

${ }^{12}$ J.-J. Greffet, M. Laroche, and F. Marquier, Phys. Rev. Lett. 105, 117701 (2010).

${ }^{13} \mathrm{We}$ assume the source to be aligned radially to an isotropic particle. For a full vectorial expression see Ref. 43.

${ }^{14}$ H. Mertens, A. F. Koenderink, and A. Polman, Phys. Rev. B 76, 115123 (2007).

${ }^{15}$ W. H. Weber and G. W. Ford, Phys. Rev. B 70, 125429 (2004); F. J. García de Abajo, Rev. Mod. Phys. 79, 1267 (2007).

${ }^{16}$ Since $\operatorname{Re} \boldsymbol{G}_{\mathrm{vac}}$ diverges at the source, and one is only interested in relative frequency shifts, it is commonly included in $\boldsymbol{\alpha}_{0}$ to yield a finite resonance frequency $\omega_{0}$. See R. Carminati, J.-J. Greffet, C. Henkel, and J. Vigoureux, Opt. Commun. 261, 368 (2006).

${ }^{17}$ Y. Xu, R. K. Lee, and A. Yariv, Phys. Rev. A 61, 033807 (2000).

${ }^{18}$ C.-T. Tai, Dyadic Green Functions in Electromagnetic Theory (IEEE, New York, 1994).

${ }^{19}$ M. Paulus, P. Gay-Balmaz, and O. J. F. Martin, Phys. Rev. E 62, 5797 (2000).

${ }^{20}$ For spherical particles of volume $V$ and dielectric constant $\epsilon(\omega)$, the electrostatic polarizability is simply $3 V(\epsilon-1) /(\epsilon+2)$. A Drude model for $\epsilon$ yields a Lorentzian. We choose $\omega_{0}=4.76 \times 10^{15} \mathrm{~s}^{-1}$ and $\gamma=8.3 \times 10^{12} \mathrm{~s}^{-1}$.

${ }^{21}$ A. F. Koenderink, Opt. Lett. 35, 4208 (2010).

${ }^{22}$ E. Prodan, C. Radloff, N. J. Halas, and P. Nordlander, Science 302, 419 (2003).

${ }^{23}$ C. F. Bohren and D. R. Huffman, Absorption and Scattering of Light by Small Particles (John Wiley and Sons, New York, 1983).

${ }^{24}$ I. Agha, J. Sharping, M. Foster, and A. Gaeta, Appl. Phys. B 83, 303 (2006).
${ }^{25}$ C. Santori, D. Fattal, and Y. Yamamoto, Single-Photon Devices and Applications (John Wiley \& Sons, New York, 2010).

${ }^{26}$ A. F. Koenderink, M. Kafesaki, B. C. Buchler, and V. Sandoghdar, Phys. Rev. Lett. 95, 153904 (2005).

${ }^{27} \mathrm{It}$ is easy to show that a small dimer antenna is described as a single scatterer with $\boldsymbol{\alpha}_{\text {eff }}=2\left[\boldsymbol{\alpha}_{\mathrm{B}}^{-1}-\boldsymbol{G}_{\mathrm{B}}\left(\boldsymbol{r}_{1}, \boldsymbol{r}_{2}\right)\right]^{-1}$.

${ }^{28}$ For a full vectorial expression of the optical theorem see Ref. 43.

${ }^{29}$ R. G. Newton, Scattering Theory of Waves and Particles (Dover, New York, 2002).

${ }^{30}$ B. C. Buchler, T. Kalkbrenner, C. Hettich, and V. Sandoghdar, Phys. Rev. Lett. 95, 063003 (2005).

${ }^{31}$ Note that $\boldsymbol{G}_{\text {vac }} \propto 1 / d^{3}$ while $\boldsymbol{G}_{\mathrm{B}}$ is of order $k^{3}$.

${ }^{32}$ M. Motsch, M. Zeppenfeld, P. W. H. Pinkse, and G. Rempe, New J. Phys. 12, 063022 (2010).

${ }^{33}$ T. Kalkbrenner, U. Håkanson, A. Schädle, S. Burger, C. Henkel, and V. Sandoghdar, Phys. Rev. Lett. 95, 200801 (2005); E. Castanié, R. Vincent, R. Pierrat, and R. Carminati, Intern. J. Opt. 2012, 452047 (2012).

${ }^{34}$ C. L. G. Alzar, M. A. G. Martinez, and P. Nussenzveig, Am. J. Phys. 70, 37 (2002).

${ }^{35}$ M. Fleischhauer, A. Imamoglu, and J. P. Marangos, Rev. Mod. Phys. 77, 633 (2005)

${ }^{36}$ B. Luk'yanchuk, N. I. Zheludev, S. A. Maier, N. J. Halas, P. Nordlander, H. Giessen, and C. T. Chong, Nat. Mater. 9, 707 (2010); M. Frimmer, T. Coenen, and A. F. Koenderink, Phys. Rev. Lett. 108 077404 (2012);

${ }^{37}$ S. Haroche, in Fundamental Systems in Quantum Optics: Proceedings of the Les Houches Summer School, Session LIII, edited by J. Dalibard, J.-M. Raimond, and J. Zinn-Justin (Elsevier, Amsterdam, 1992).

${ }^{38}$ M. L. Andersen, S. Stobbe, A. S. Sørensen, and P. Lodahl, Nat. Phys. 7, 215 (2011).

${ }^{39}$ L. Verslegers, Z. Yu, Z. Ruan, P. B. Catrysse, and S. Fan, Phys. Rev. Lett. 108, 083902 (2012).

${ }^{40}$ A. Mazzei, S. Götzinger, L. de S. Menezes, G. Zumofen, O. Benson, and V. Sandoghdar, Phys. Rev. Lett. 99, 173603 (2007).

${ }^{41}$ Y. D. Wilde, F. Formanek, R. Carminati, B. Gralak, P.-A. Lemoine, K. Joulain, J.-P. Mule, Y. Chen, and J.-J. Greffet, Nature (London) 444, 740 (2006); X. Liu, T. Tyler, T. Starr, A. F. Starr, N. M. Jokerst, and W. J. Padilla, Phys. Rev. Lett. 107, 045901 (2011); S.-Y. Lin, J. G. Fleming, and I. El-Kady, Opt. Lett. 28, 1909 (2003); S. E. Han and D. J. Norris, Phys. Rev. Lett. 104, 043901 (2010).

${ }^{42}$ F. Vollmer and S. Arnold, Nat. Methods 5, 591 (2008).

${ }^{43}$ J. E. Sipe and J. V. Kranendonk, Phys. Rev. A 9, 1806 (1974). 